\title{
Applicability and Performance of Heart Rate Variability Methods
}

\author{
J Haaksma, J Brouwer, WA Dijk, MP van den Berg, F Takens, WRM Dassen* \\ University of Groningen, *University of Maastricht, The Netherlands
}

\begin{abstract}
Heart Rate Variability (HRV) may be measured using different measurement techniques. Almost all of these techniques deal with ectopic beats. Either these beats are excluded from the analysis or they are required. To access the functional character and easiness of use of several HRV techniques we compared Holter registrations in three patient groups: normal subjects, post infarction patients and patients with heart failure. Analysis speed, sensitivity to non sinus intervals, applicability and accuracy were investigated. Time as well as frequency domain techniques, heart rate turbulence and a non linear measure of HRV were studied. The results of the analysis suggest that non linear analysis is as least as potent as conventional HRV techniques in discriminating between patient groups. This technique can be applied in all patients with minimal computational effort and without the need for accurate analysis of the ECG.
\end{abstract}

\section{Introduction}

For risk stratification a method that can be applied to a group as a whole offers significant advantages to a technique that can only be applied in a specific subset. Many heart rate variability (HRV) techniques that are used nowadays can only be applied to a small subsection of a certain population. The main cause for this is the existence of ectopic data and noise. Spectral analysis requires a signal with as little ectopics as possible, while heart rate turbulence (HRT) requires the presence of ectopic beats. According to the "standards of measurements" (Circulation 1996) [7] the calculation of spectral analysis "should preferably be done on short segments which are free of ectopy". Requiring the presence or absence of ectopics however, causes a significant selection bias. Non linear measures of HRV such as correlation integral, dimension of the signal and entropy may provide an alternative to the linear techniques. Since the prevalence of ectopic beats is influenced by the activity of autonomic nervous system, it might be argued that the signal as a whole, i.e. including all beats is also a reflection of the autonomic state. However, because of the nature of such computations the use of such a technique over an entire Holter recording is extremely time-consuming. We therefore investigated the possibility to accelerate nonlinear analysis using a limited amount of data.

\section{Methods}

To assess the functional character and easiness of use of several HRV methods we compared Holter registrations in three patient groups: I. normal subjects (n $=196)$, II: post infarction patients $(n=208)$ and III: patients with moderate heart failure $(n=170)$. Analysis speed, sensitivity to noise, applicability and accuracy were investigated. All recordings were carefully analyzed by an experienced analyst using a GE Marquette Mars 8000 Holter system. After analysis the RR interval data was exported and analyzed using custom developed software (COHWIN). This software package is written in Delphi ${ }^{\circledR}$ and focuses especially on signal analysis.

\subsection{Time domain analysis}

Time domain analysis was performed using normal to normal (NN) intervals only. The average NN interval (AVGNN) the standarddeviation (SDNN) and the root mean square of successive differences (rMSSD) were determined.

\subsection{Frequency domain analysis}

The analysis was performed over 5 minutes segments using Discrete Fourier Transformation [1-5]. The advantages of this method over fast fourier transformation have been described before[2]. Only data segments containing less than $5 \%$ noise or ectopy were accepted for analysis. This is a percentage based on time, not number of beats [6]. Finally a check was performed to ensure the validity of the spectral analysis by comparing the square root of the overall spectral power to SDNN. ( square root (total frequency power) / SDNN) $\mathrm{x}$ $100 \%$. Parsevals theorem states that the power in time domain is equal to the power in frequency domain, therefore the result of this computation should be $100 \%$. This index is called the Paseval index. Deviations of the 
Paseval index may be caused by non stationarity. All deviations $>10 \%$ were also excluded from further analysis.

\subsection{Heart Rate Turbulence}

HRT measures the effect of a premature ventricular complex (PVC) on the sinus rhythm. In healthy individuals a PVC will show an increase in sinusrate as a consequence of the change in bloodpressure. In diseased patients this reaction will be blunted[8]. The original article that described HRT does not recommend a minimum number of PVC's to be present. In a training sample the authors use a minimum of 240 PVC's per recording while in the patient populations all recordings that contain PVC's seem to be used. Two variables are distinguished: turbulence onset (TO) and turbulence slope (TS). TO is defined as the difference between the average of two intervals after a PVC and the two intervals preceding the PVC. TS is defined as the maximum positive slope of a regression line over a sequence of five consecutive sinus RR intervals, during the first twenty intervals after a PVC. To study the effect of the PVC's we determined these HRT variables for both recordings containing > 240 PVC's (TO240 and TS240) and for recordings that contained at least one PVC (TOall and TSall).

\subsection{Non linear analysis}

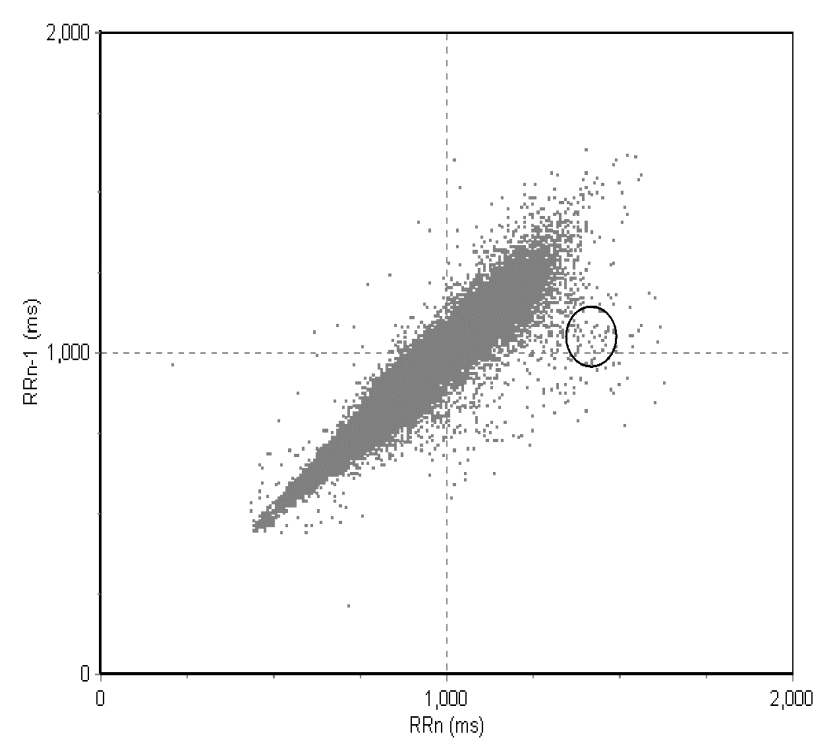

Figure 1: A plot of $R R_{n}$ versus $R_{R}-1$. The circle represents the threshold $(\varepsilon)$ in which the number of correlation hits are counted. Since here two intervals $\left(R R_{n}\right.$ and $\left.R R_{n-1}\right)$ are used we speak of an embedding dimension of two.
In order to compute the correlation index of a signal, all possible interval sequences are determined and compared to all other possible sequences. This computation is repeated for several embedding dimensions. When the difference is below a threshold, a "correlation hit" is counted. By varying the threshold $(\varepsilon)$, a function of correlation versus the threshold is obtained. The correlation index (CI) is 1 when all interval sequences differ less than the threshold from all other interval sequences. In a formula this is described as:

$$
\begin{aligned}
& C_{(\varepsilon)}=\frac{2}{N(N-1)} \sum_{i=1}^{N} \sum_{j=i+1}^{N} \Theta\left(\varepsilon-\left\|x_{i}-x_{j}\right\|\right) \\
& \text { where } \Theta_{(x)}=0 \text { if } x \leq 0 \text { and } \Theta_{(x)}=1 \text { if } x>0 .
\end{aligned}
$$

Non linear analysis is a computational very demanding technique. For example, in order to calculate the index at nine embedding dimensions $(m=2-10)$, decreasing the value of the threshold $\varepsilon$ in 20 steps, a Holter that contains around 100.000 intervals will take $1.8 * 10^{12}$ comparisons. $(9 * 20 * 100.000 * 100.000)$ Even on a fast PC ( Pentium IV, 2 Ghz ) this takes a very long time of approximately 2 days. In order to increase analysis speed we used an algorithm the utilizes 100.000 randomly selected interval combinations. The results of such an algorithm yields nearly identical results compared to the analysis of the full signal.

\section{Results}

\begin{tabular}{llll}
\hline & I & II & III \\
\hline n (patients) & 196 & 208 & 170 \\
AVG PVC's & 32 & 144 & 291 \\
Zero PVC's & $76(39 \%)$ & $17(9 \%)$ & $6(3 \%)$ \\
$>240$ PVC's & $6(3 \%)$ & $27(12 \%)$ & $74(40 \%)$ \\
\hline
\end{tabular}

Table 1 : Patient characteristics.

The patient characteristics are shown in table I. The average number of PVC's per recording, the number of patients with $>240$ PVC per recording and the number of patients that had no PVC's. PVC's are most frequent in group III (CHF patients) while, as expected, these were relatively infrequent in normal subjects. Nevertheless PVC's were present in $61 \%$ of the normal subjects. Only $3 \%$ of the normal subjects had $>3 \%$ PVC's. In the CHF group this turned out to be true in $40 \%$ of the patients. 


\subsection{Time and frequency domain}

Table 2 shows the results of the time and frequency domain analysis. All techniques (time, frequency domain as well as HRT) show lower HRV in the patient categories compared to the normal subjects. The results of the HRT analysis also depend on the number of PVC's that is required to perform the analysis.

\begin{tabular}{llll}
\hline Group & I & II & III \\
\hline AVGNN & 809 & 947 & 759 \\
SDNN & 153 & 141 & 118 \\
rMSSD & 48 & 53 & 34 \\
TP & 5522 & 5259 & 2607 \\
LF & 1465 & 1065 & 548 \\
HF & 568 & 583 & 208 \\
& & & \\
TO240 & -4.92 & -2.23 & -1.30 \\
TS240 & 4.04 & 4.93 & 1.27 \\
TOall & -3.73 & -2.03 & -1.46 \\
TSall & 7.02 & 3.49 & 1.88 \\
\hline
\end{tabular}

Table 2: Time, frequency domain and heart rate turbulence for the three groups. The TO240 and TS240 are calculated over recordings containing $>240$ PVC's while the TOall and TSall are obtained from registrations with at least one PVC.

\subsection{Nonlinear analysis}

The result of a CI calculation in a normal subject is shown in figure 2 .

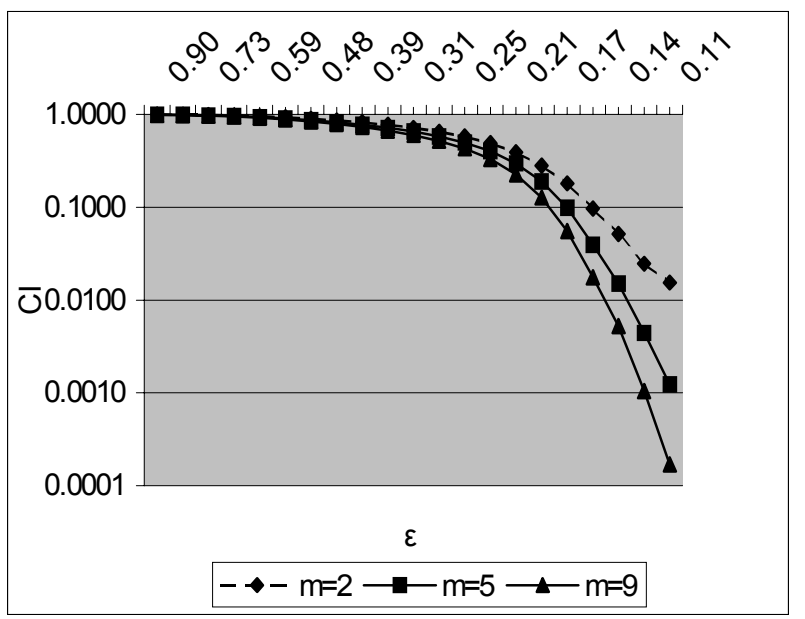

Figure 2: CI for three different embedding dimensions $(\mathrm{m}=2,5$ and 9). CI is shown on the $\mathrm{Y}$ axis and is represented as a $\log$ scale. The $\mathrm{X}$-axis shows the value of the threshold $(\varepsilon)$, where 1 represents the average RR interval.
From figure 2 we can see that larger embedding dimensions show less correlation than small embedding dimensions. The difference between the various embedding dimensions increase for lower values of $\varepsilon$.

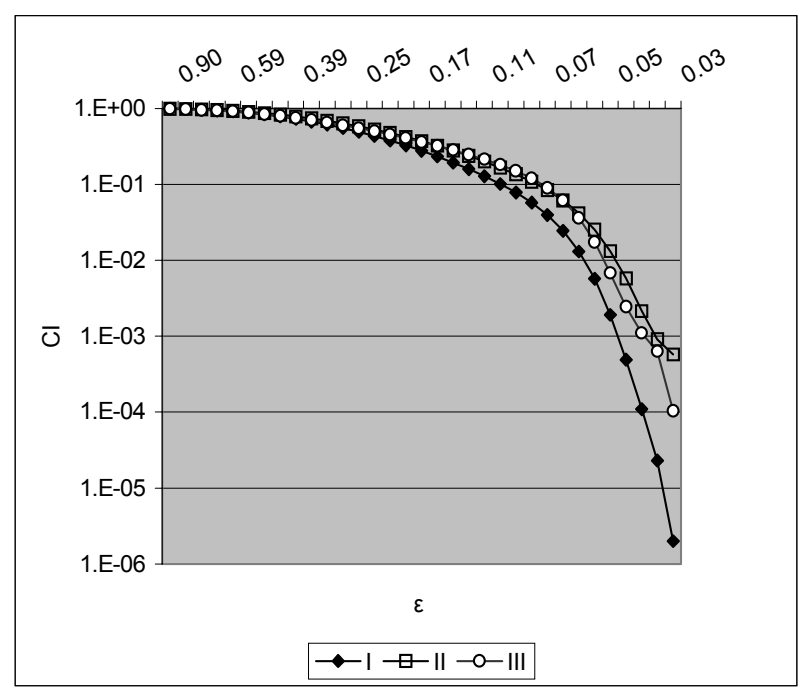

Figure 3: the CI for all the three patient groups calculated at an embedding dimension of 9. The CI is presented on the $\mathrm{Y}$-axis (log scale) while the value of $\varepsilon$ is on the $\mathrm{X}$-axis.

When we look at the different patient categories we see a the lowest degree of correlation in the normal population (see figure 3) indicating more irregular rhythm compared to both patient groups. The difference between the normal subjects and the two patient groups is shown in table 3.

\begin{tabular}{|l|l|l|l|l|}
\hline \multirow{2}{*}{$\begin{array}{l}\text { group } \Rightarrow \\
\text { threshold } \Downarrow\end{array}$} & \multicolumn{2}{|c|}{ II } & \multicolumn{2}{c|}{ III } \\
\cline { 2 - 5 } & $\mathrm{m}=4$ & $\mathrm{~m}=9$ & $\mathrm{~m}=4$ & $\mathrm{~m}=9$ \\
\hline $50 \%$ & 1 & 0 & 3 & 3 \\
\hline $25 \%$ & 9 & 11 & 15 & 18 \\
\hline $12.5 \%$ & 28 & 55 & 30 & 49 \\
\hline $6.25 \%$ & 55 & 152 & 70 & 151 \\
\hline
\end{tabular}

Table 3: percentage of difference between the patient groups and normal subjects. Again the threshold is shown as a percentage of the average RR interval.

\section{Discussion}

The conventional HRV techniques (time and frequency domain measures of HRV) show different average values for the various groups. These techniques however require accurate editing and correction of the Holter data. The fact that the average heart rate of the infarction group is lower compared to the normal 
population is explained by the fact that betablockers are virtually a standard treatment after myocardial infarction. While HRT also yields different discriminating results for the populations that were studied, it also calls for accurate analysis of the ECG. Limitation of this technique is that due to the lack of PVC's it cannot be applied in every patient. For example: requiring $>240$ PVC's leads to exclusion of $88 \%$ of the post infarction patients. Selecting patients based on the number of PVC's will cause a severe selection bias.

Non linear analysis shows the largest differences between the different patient groups. The results show that large values of the embedding dimension and smaller values of $\varepsilon$ are the best option to discriminate between the groups. More importantly non linear analysis can be used without the need for accurately distinguishing between normal and ectopic beats. As a result it can be applied in all patients. Normally this technique does not present a practical alternative, since the it is an extremely time consuming procedure. However using only a limited number of interval combinations decreases the computational time to just a matter of seconds.

The editing that is required and the selection bias introduced by routine HRV and HRT methods justifies the development of more robust (non-linear) techniques.

\section{Acknowledgement}

The financial support of the Dutch Heart foundation (grant number 1999B119) is greatly acknowledged.

\section{References}

[1] Mulder et al. Information processing and cardiovascular control. Psychophysiology 1981; 14: 392-402

[2] Rompelman et al. The measurement of heart rate variability spectra with the help of a personal computer. IEEE Trans. on Biom. Eng., vol. BME-29, no 7 Jul. 1982

[3] De Boer et al. Comparing spectra of a series of point events particularly for heart rate variability data. Trans. on Biom. Engineering, vol. BME-31, no 4 April 1984

[4] Mulder et al. CARSPAN, A spectral analysis program for cardiovascular time series. Computers in Psychology: Methods, Instrumentation \& Psychodiagnostics., 1988 (pp. 30-38). Lisse: Swets \& Zeitlinger

[5] Mulder, L.J.M. Assessment of cardiovascular reactivity by means of Spectral Analysis. 1988 Ph.D. Thesis, Groningen, University of Groningen.

[6] Haaksma et al. Effects of automatic ectopy exclusion on the analysis of heart rate variability using a percentile exclusion rule. Computers in Cardiology 1995;197-200 Los Almalitos: IEEE Computers Society Press

[7] Task force of the European Society of Cardiology and the North American Society of Pacing and Electrophysiology Heart Rate Variability, Standards of measurement, physiological interpretation and clinical use. Circulation Vol 1996; 93;5: 1043-1065

[8] Schmidt G, Malik M, Barthel P, Schneider R, Ulm K, Rolnitzky L, Camm AJ, Bigger Jr. JT, Schömig A. Heartrate turbulence after ventricular premature beats as a predictor of mortality after acute myocardial infarction. Lancet 1999; Vol. 353; No. 9162; 1390-96.

Address for correspondence:

\author{
Dr. J. Haaksma \\ Thoraxcenter C0.131 \\ University Hospital Groningen \\ Postbus 30.001 \\ 9700 RB Groningen \\ The Netherlands \\ Fax: +31503611470 \\ Email address J.Haaksma@thorax.azg.nl
}

